\title{
Éditorial
}

\section{L'agriculture intensive devra connaître des changements inévitables}

\section{Michel Griffon}

Conseiller pour le développement durable auprès du directeur général du Cirad

Centre de coopération internationale en recherche agronomique pour le développement (Cirad) 4bis, avenue de la Belle-Gabrielle, 94736 Nogent-sur-Marne cedex <michel.griffon@cirad.fr>

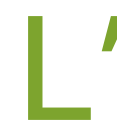

agriculture intensive en énergie et en intrants chimiques devra connaitre des changements dont on peut maintenant penser qu'ils deviennent inévitables. Un faisceau de contraintes nouvelles se prépare à atteindre directement toutes les agricultures fortement utilisatrices d'engrais, de produits phytosanitaires et de carburants fossiles, qu'il s'agisse des agricultures européennes, nord-américaines, de celles des grandes exploitations des pays exportateurs comme le Brésil, l'Argentine, l'Australie, ou des agricultures de révolution verte en particulier en Asie. Quelles en sont les causes?

La première cause est l'augmentation des prix du pétrole en raison de sa raréfaction dans les décennies qui viennent. L'agriculture conventionnelle qui repose sur le labour utilise pour cette opération des quantités importantes d'énergie fossile. Dans un contexte de concurrence internationale sur les exportations avivé par les débats à l'Organisation mondiale du commerce (OMC), le travail du sol devient un poste de dépense trop lourd Le travail du sol simplifié et le semis direct vont sans doute s'imposer partout où cela est possible.

La deuxième cause est le coût des engrais chimiques. Les engrais azotés sont issus du gaz naturel dont le prix suit celui du pétrole, et leur production nécessite des quantités importantes d'énergie. Leur prix devrait donc lui aussi augmenter. Par ailleurs, les phosphates sont aussi tributaires de l'énergie dont il faut aussi d'importantes quantités pour les extraire et les produire. Leur coût de transport devrait être plus cher et le transport est un poste de charge important dans le prix : il faut les transporter jusqu'aux usines, puis aux ports, les charger sur des bateaux et les transporter, les décharger, les acheminer jusqu'aux exploitations et les épan- dre. Il faut aussi signaler que le phosphate est une ressource fossile dont on estime que les réserves les plus faciles à extraire et à transformer pourraient ne pas durer plus longtemps que le pétrole. Certes, les autres réserves pourraient durer encore quelques siècles mais elles sont plus difficiles à extraire : leur coût de production sera donc sans doute de plus en plus élevé. Ces différentes causes devraient amener à reconsidérer les bases de la fertilité des sols. Cela requiert d'ores et déjà des investissements scientifiques importants.

Une troisième cause de changement est liée à l'opposition de plus en plus résolue de la société aux pesticides, en particulier aux insecticides, fongicides et herbicides utilisés dans les grandes cultures. La littérature scientifique commence à mettre en cause différentes molécules dans des maladies humaines, et la presse donne à penser par exemple que les pesticides pourraient être à l'origine d'un grand nombre de cancers. Les pesticides font aussi l'objet d'attaques concernant leur toxicité environnementale, par exemple vis-à-vis de certains auxiliaires des cultures. Enfin, les molécules pesticides semblent devenir rapidement obsolètes en raison des résistances génétiques des insectes, champignons microscopiques ou adventices acquises par mutation. Les industries phytosanitaires voient ainsi leur activité potentiellement fragilisée, à telle enseigne que l'on doit maintenant s'interroger sur les solutions alternatives aux molécules actuelles et même à la lutte chimique dans son ensemble.

Ces événements actuels et les risques potentiels accélèrent l'avènement de ce que l'on a appelé la "révolution doublement verte". D'autres termes presque synonymes sont maintenant largement connus : agriculture de conservation, agroécologie, écoagriculture, par exemple. Le mouvement semble 
maintenant en marche et de nombreux exemples concrets sont aujourd'hui disponibles. En France, des groupes de producteurs expérimentent eux-mêmes des pratiques nouvelles de semis direct et de lutte intégrée contre les maladies et les ravageurs. La grande diversité de ces initiatives pourrait rapidement déborder les capacités de la recherche à faire des expérimentations de contrôle et de validation technique. Il faut se réjouir de l'existence d'un tel mouvement après qu'il a pu être observé non seulement au Brésil, en Argentine, chez des petits producteurs d'Amérique centrale, mais aussi maintenant en Inde.

Cependant d'autres changements interviennent, non pas seulement dans le domaine technique mais aussi dans le domaine plus directement économique. L'accroissement rapide de la demande en biocarburants liquides partout dans le monde crée une concurrence immédiate pour l'utilisation des sols entre la finalité alimentaire et la finalité énergétique. Cela crée rapidement une nouvelle rareté de la terre dont il faut observer les conséquences. Il serait grave que cela entraîne dans certaines régions du monde une nouvelle course à la terre et à la déforestation. Il faut surveiller l'évolution des prix de la terre qu'une nouvelle rareté pourrait pousser à la hausse, limitant dans beaucoup de régions la capacité à redistribuer les terres pour les plus pauvres. Il faut aussi surveiller l'évolution des prix agricoles destinés à l'alimentation : la presse internationale a largement rendu compte du fait que la substitution rapide de la production de biocarburants à la production de maïs aux États-Unis a réduit les exportations de cette céréale vers le Mexique (dont la libéralisation de l'économie agricole avait privilégié les importations de maïs face à la production locale) et en a accru considérablement le prix alors qu'il s'agit d'un produit de base, presque mythique, dans l'alimentation mexicaine. On pourrait de même s'attendre à une rareté relative du blé faisant monter les prix internationaux encore plus que depuis deux ans. Si, par extraordinaire, une plus grande variabilité climatique accroissait la fréquence des sécheresses ou des périodes de fortes intempéries chez les pays producteurs (on pense à la sécheresse d'Australie de 2005 et 2006), alors la dynamique des prix et des marchés internationaux de céréales serait changée. De nombreux scénarios sont possibles, qu'il faudra analyser dans les temps qui viennent. L'un d'entre eux pourrait être favorable aux agriculteurs des pays en développement importateurs de céréales, en particulier en Afrique : si les prix des céréales augmentaient durablement, rompant ainsi historiquement avec une tendance centenaire, ce serait alors en effet une occasion historique à saisir pour que la production locale redevienne compétitive par rapport aux importations et qu'elle puisse offrir un espace de croissance solide aux agricultures locales.

Mais d'autres conséquences pourraient être attendues du côté de la politique agricole commune (PAC) et des négociations entreprises dans le cadre de l'OMC. Le point de blocage principal des négociations tient depuis longtemps aux subventions à l'agriculture que consentent l'Europe et les États-Unis, qui sont de plus en plus accusés de compenser par ces subventions le différentiel entre les prix céréaliers internationaux et leurs prix intérieurs. Une hausse des prix internationaux réduirait mécaniquement l'importance du problème et limiterait les tensions dans la négociation. Par ailleurs, l'Europe pourrait consacrer les sommes libérées au financement de la mutation technologique que l'agriculture devra opérer.

Sur toutes ces questions, dans les années qui viennent la recherche a un rôle majeur d'éclairage de la décision publique. 